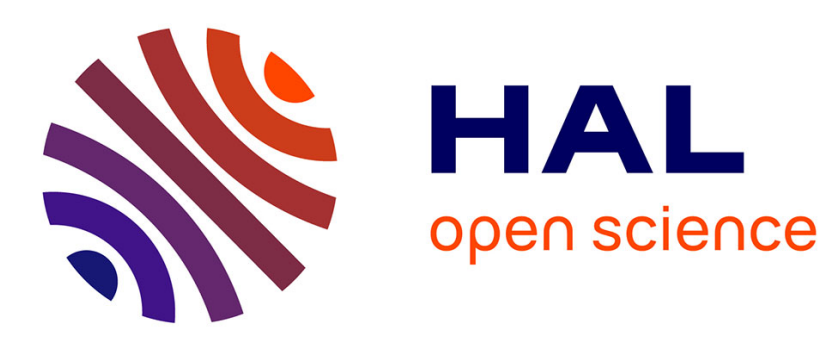

\title{
Dynamic modeling of cooperative robots holding flexible objects
}

\author{
Philip Long, Wisama Khalil, Philippe Martinet
}

\section{To cite this version:}

Philip Long, Wisama Khalil, Philippe Martinet. Dynamic modeling of cooperative robots holding flexible objects. ICAR 2015 - International Conference on Advanced Robotics, Jul 2015, Istanbul, France. pp.182-187, 10.1109/ICAR.2015.7251453 . hal-02459380

\section{HAL Id: hal-02459380 \\ https://hal.inria.fr/hal-02459380}

Submitted on 29 Jan 2020

HAL is a multi-disciplinary open access archive for the deposit and dissemination of scientific research documents, whether they are published or not. The documents may come from teaching and research institutions in France or abroad, or from public or private research centers.
L'archive ouverte pluridisciplinaire HAL, est destinée au dépôt et à la diffusion de documents scientifiques de niveau recherche, publiés ou non, émanant des établissements d'enseignement et de recherche français ou étrangers, des laboratoires publics ou privés. 


\title{
Dynamic Modeling of Cooperative Robots holding Flexible Objects
}

\author{
Philip Long, Wisama Khalil and Philippe Martinet
}

\begin{abstract}
In this paper a method of calculating the inverse and direct dynamic model of cooperative manipulators handling flexible objects is presented. In order to solve the dynamics of this system, the closed chain is divided into two subsystems, one is flexible, representing the object and the other is rigid, representing the tree structure of the two manipulators. The two subsystems are connected by calculating the wrench transmitted by the end effectors. An analytic solution is obtained for the dynamic model. The proposed solution leads to a classification of objects grasped by multiple manipulators.
\end{abstract}

\section{INTRODUCTION}

The accurate control of flexible objects by robots is an interesting subject due to its industrial importance, notably in the medical [1] and food processing [2] sectors. The proposed solutions dealing with object flexibility are into two classes, sensor based [3], [4] and model based [5], [6]. In this work, we focus on model based solutions, where for closed chain cooperative robots research has been typically approached from an object oriented viewpoint. Cooperative robots can be considered as redundantly actuated systems since all the degrees of freedom of each manipulator are motorized. Thus the system can achieve secondary goals, such as load distribution [7], backlash elimination [8] and optimization of independent actuators [9]. In addition to this, the object's internal state can be controlled. Depending on the object type and the grasp structure, the internal variables may represent the object's internal loading [10], shape [11], [12] or vibration [5].

For systems manipulating rigid objects, the controlled states are divided into position and force variables. This has led to the adoption of classical force control schemes for cooperative systems. For example, hybrid position/force schemes are proposed in [13], [14] while impedance controllers are proposed in [10], [15]. If the object is fully flexible, the redundant actuators can be used to maintain the vibrations at an acceptable level. For example, in [16], [17], vibration suppression control schemes for a dual-arm system handling a beam are proposed. If the object is flexible in some directions but not in others [5], [18], both the vibration and internal forces must be taken into account as secondary control objectives. Objects of limited flexibility, where the manipulators are capable of directly controlling these variables, are said to be articulated. In this case, the redundant actuation can be used to control the object's state by using classical rigid body modeling techniques [19]. Alternatively, using lumped parameter methods [12], [20], the spring compression variables can be controlled during the object motion.

We propose a general modeling strategy that allows the identification and resolution of the above cases. The modeling

\footnotetext{
*This work is supported by the ARMS project, French grant ANR-10-SEGI008 .

Authors are with the École Centrale de Nantes and IRCCyN, 44321 Nantes France (e-mail: firstname.lastname@irccyn.ec-nantes.fr).
}

strategy decouples the closed chain system into its rigid and flexible subsystems [6]. The derivation is carried out using distributed flexibilities i.e. modal analysis [21]. The subsystems are linked together by the wrench applied at the end effector's grasp.

The principal contribution of this paper is the derivation of the inverse and direct dynamic model of a general multi-arm system grasping a common object. The obtained solution is closed form, which is computationally more efficient than its numerical counterparts and can be exploited in the derivation of dynamic control laws. The equations give an insight into the object type. In contrast to previous works, the proposed algorithm can resolve diverse objects: rigid, articulated and objects whose flexibility can be represented by modal distribution, leading to increased modularity in potential applications. Three corresponding cases studies with solutions are presented and compared with a commercial simulator.

The layout of the paper is as follows. In Section II, the dynamic modeling of a flexible free body is recalled. In Section III the general dynamic equation for a cooperative system handling a flexible object is derived. The resolution of this equation and identification of the type of grasped object is given in Section IV. The conclusion to this work and possible areas of future research are given in Section V.

\section{GENERALIZED NEWTON-EULER MODEL}

This section recalls the modeling procedure for the flexible body. The object's flexibility is modeled using the generalized Newton-Euler model [21], [22]. In this model, the NewtonEuler formalism is used to describe the rigid body motions while the Lagrangian formalism is used to describe its elastic motions. The main assumption in this formalism is that the flexible body motion can be approximated by the sum of the rigid body motions and the flexible body deformations.

\section{A. Kinematic Modeling of a flexible body}

The position, $\mathbf{p}_{i}$ of any point $i$ of the flexible body is obtained by the sum of the position of the body's reference

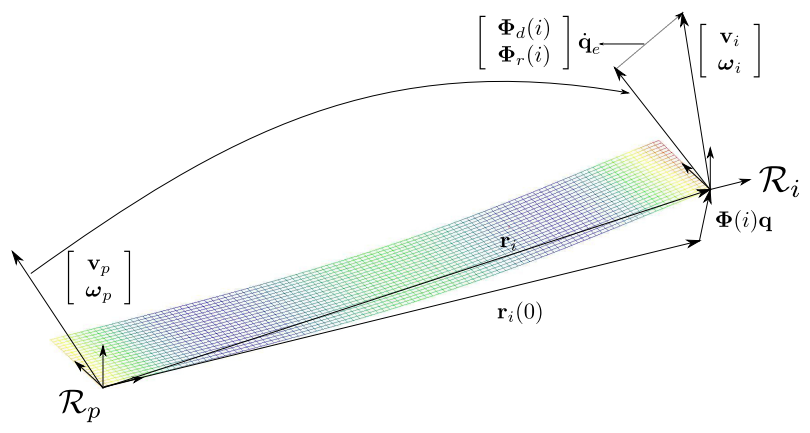

Fig. 1. Principle of Modal Superposition 
frame, denoted as $\mathbf{p}_{p}$, and the position vector $\mathbf{r}_{i}$ as shown in Fig.1.

$$
\mathbf{p}_{i}=\mathbf{p}_{p}+\mathbf{r}_{i}
$$

$\mathbf{r}_{i}$ is the position vector from frame $\mathcal{R}_{p}$ to frame $\mathcal{R}_{i}$ consisting of the corresponding vector for the rigid body, denoted as $\mathbf{r}_{i}(0)$ and the deformation at point $i$ :

$$
\mathbf{r}_{i}=\mathbf{r}_{i}(0)+\sum_{k=1}^{N} \boldsymbol{\Phi}_{d k}(i) q_{e k}
$$

$\mathbf{q}_{e}=\left(q_{e 1} \ldots q_{e k} \ldots q_{e N}\right)$, is the $N \times 1$ vector of generalized elastic coordinates while $\dot{\mathbf{q}}_{e}$ and $\ddot{\mathbf{q}}_{e}$ are the vectors of velocity and acceleration, respectively. By obtaining the derivative of (2):

$$
\dot{\mathbf{r}}_{i}=\boldsymbol{\omega}_{p} \times \mathbf{r}_{i}+\sum_{k=1}^{N} \boldsymbol{\Phi}_{d k}(i) \dot{q}_{e k}
$$

$\boldsymbol{\omega}_{p}$ is defined as the vector of angular velocity of the moving object. Equation (3) gives the change in the relative position of point $i$. The kinematic twist of the flexible body at point $i$ is given as:

$$
\begin{aligned}
{\left[\begin{array}{c}
\mathbf{v}_{i} \\
\boldsymbol{\omega}_{i}
\end{array}\right] } & =\left[\begin{array}{cc}
\mathbf{I}_{3} & -\hat{\mathbf{r}}_{i} \\
\mathbf{0}_{3} & \mathbf{I}_{3}
\end{array}\right]\left[\begin{array}{c}
\mathbf{v}_{p} \\
\boldsymbol{\omega}_{p}
\end{array}\right]+\left[\begin{array}{l}
\boldsymbol{\Phi}_{d}(i) \\
\boldsymbol{\Phi}_{r}(i)
\end{array}\right] \dot{\mathbf{q}}_{e} \\
\boldsymbol{\Phi}_{d}(i) & =\left[\begin{array}{llll}
\Phi_{d 1}(i) & \Phi_{d 2}(i) & \ldots & \Phi_{d N}(i)
\end{array}\right] \\
\boldsymbol{\Phi}_{r}(i) & =\left[\begin{array}{llll}
\Phi_{r 1}(i) & \Phi_{r 2}(i) & \ldots & \Phi_{r N}(i)
\end{array}\right]
\end{aligned}
$$

which becomes

$$
\mathbf{V}_{i}={ }^{i} \mathbf{S}_{p} \mathbf{V}_{p}+\boldsymbol{\Phi}(i) \dot{\mathbf{q}}_{e}
$$

where $\boldsymbol{\Phi}(i)=\left[\begin{array}{ll}\boldsymbol{\Phi}_{d}^{T}(i) & \boldsymbol{\Phi}_{r}^{T}(i)\end{array}\right]^{T}$.

The shapes functions are divided by position, denoted as $\boldsymbol{\Phi}_{d k}$ and orientation denoted as $\boldsymbol{\Phi}_{r k} \cdot{ }^{i} \mathbf{S}_{p}$ is the $(6 \times 6)$ screw transformation matrix from frame $p$ to frame $i . \mathbf{v}_{p}$ is defined as the component of rigid velocity of the object, while $\dot{\mathbf{v}}_{p}$ and $\dot{\boldsymbol{\omega}}_{p}$ denote the linear and angular acceleration. $\mathbf{V}_{i}$ denotes the kinematic screw evaluated at point $i$. The forces and moments, denoted as $\mathbf{f}_{i}$ and $\mathbf{n}_{i}$, applied to point $i$ are transformed to the flexible body's origin by:

$$
\left[\begin{array}{c}
\mathbf{f}_{p} \\
\mathbf{n}_{p} \\
\mathbf{Q}_{p}
\end{array}\right]=\left[\begin{array}{c}
{ }^{i} \mathbf{S}_{p}^{T} \\
\boldsymbol{\Phi}^{T}(i)
\end{array}\right]\left[\begin{array}{c}
\mathbf{f}_{i} \\
\mathbf{n}_{i}
\end{array}\right]
$$

where $\mathbf{Q}_{p}$ denotes the generalized elastic forces.

\section{B. Dynamic Modeling of a flexible body}

The flexible body's dynamic model is obtained using the principle of virtual powers [21] as:

$$
\begin{aligned}
& {\left[\begin{array}{c}
\mathbf{f}_{p} \\
\mathbf{n}_{p} \\
\mathbf{Q}_{p}
\end{array}\right]=\left[\begin{array}{ccc}
m \mathbf{1}_{3} & \mathbf{M} \hat{\mathbf{S}}_{r}^{T} & \mathbf{M S}_{d e} \\
\mathbf{M} \hat{\mathbf{S}}_{r} & \mathbf{I}_{0 p} & \mathbf{M S _ { r e }} \\
\mathbf{M S}_{d e}^{T} & \mathbf{M S}_{r e}^{T} & \mathbf{m}_{e e}
\end{array}\right]\left[\begin{array}{c}
\dot{\mathbf{v}}_{p}-\mathbf{g} \\
\dot{\boldsymbol{\omega}}_{p} \\
\ddot{\mathbf{q}}_{e}
\end{array}\right]} \\
& +\left[\begin{array}{c}
\mathbf{f}_{c} \\
\mathbf{n}_{c} \\
\mathbf{Q}_{c}
\end{array}\right]+\left[\begin{array}{c}
0 \\
0 \\
\mathbf{K}_{e e} \mathbf{q}_{e}+\mathbf{D}_{e e} \dot{\mathbf{q}}_{e}+\boldsymbol{\lambda}_{f}
\end{array}\right]
\end{aligned}
$$

where $m$ denotes mass of the body, while the first moments of inertia are defined as $\mathbf{M} \hat{\mathbf{S}}_{r}^{T}$. The total inertia tensor of the body is defined as $\mathbf{I}_{0 p}$. $\mathbf{M S} \mathbf{S}_{d e}$ is a $(3 \times N)$ matrix. $\mathbf{M S} \mathbf{S}_{r e}$ is a $(3 \times N)$ matrix. $\mathbf{m}_{e e}$ is the $(N \times N)$ elastic inertia matrix. The centrifugal and Coriolis forces, moments and generalized elastic forces are given by $\mathbf{f}_{c}, \mathbf{n}_{c}$ and $\mathbf{Q}_{c}$ respectively. The generalized stiffness and damping are given by $\mathbf{K}_{e e}$ and $\mathbf{D}_{e e}$. Finally, $\boldsymbol{\lambda}_{f}$ are elastic forces generated by the grasping constraints and $\mathbf{g}$ is the gravity vector. The full derivation of these terms is found in [21].

\section{DyNAMiC MOdel OF COOPERATIVE MANipulators HANDLING FLEXIBLE OBJECTS}

The procedure is outlined for a system of $n$ non-redundant serial arms, composed of rigid links, grasping a common object. The $i^{t h}$ arm, $i=1 \ldots n$,

- contains $a_{i}$ actuated joints and $m_{i}$ movable links.

- $\quad$ transmits a wrench of dimension $c_{i}$ to the object $(1 \leq$ $\left.c_{i} \leq 6\right)$.

We define:

$$
a=\sum_{i=1}^{n} a_{i} \quad m=\sum_{i=1}^{n} m_{i} \quad c=\sum_{i=1}^{n} c_{i}
$$

The object is defined by $N$ flexible coordinates (the dimension of the modal representation of the flexibility).

\section{A. Rigid Arm Equations}

The $\left(m_{i} \times 1\right)$ vectors of joint positions, velocities, accelerations and torques of arm $i$ are denoted as $\mathbf{q}_{i}, \dot{\mathbf{q}}_{i}, \ddot{\mathbf{q}}_{i}$ and $\boldsymbol{\Gamma}_{i}$ respectively. The $\left(c_{i} \times 1\right)$ vectors of velocity and acceleration at grasping point $i$, representing the terminal point of arm $i$, are defined as:

$$
\mathbf{V}_{i}=\mathbf{J}_{i} \dot{\mathbf{q}}_{i} \quad \dot{\mathbf{V}}_{i}=\mathbf{J}_{i} \ddot{\mathbf{q}}_{i}+\dot{\mathbf{J}}_{i} \dot{\mathbf{q}}_{i}
$$

where $\mathbf{J}_{i}\left(c_{i} \times m_{i}\right)$ is the kinematic Jacobian of arm $i$. Furthermore, for each arm the following quantities must be calculated: the inertia matrix $\mathbf{A}_{i}\left(m_{i} \times m_{i}\right)$ and $\mathbf{c}_{i}$ the $\left(m_{i} \times 1\right)$ term representing the Coriolis, centrifugal and gravity torques.

Let us define $\mathbf{A}_{x i}$, a $\left(c_{i} \times c_{i}\right)$ matrix, and $\mathbf{c}_{x i}$, a $\left(c_{i} \times 1\right)$ vector, as the robots inertia matrix and the vector of Coriolis, centrifugal and gravity torques transformed to the Cartesian space at the grasp point $i$ respectively [23]:

$$
\mathbf{A}_{x i}=\mathbf{J}_{i}^{-T} \mathbf{A}_{i} \mathbf{J}_{i}^{-1} \quad \mathbf{c}_{x i}=\mathbf{J}_{i}^{-T} \mathbf{c}_{i}-\mathbf{A}_{x i} \dot{\mathbf{J}}_{i} \dot{\mathbf{q}}_{i}
$$

The kinematic Jacobian matrix relates the joint torques of robot $i$ to the wrench transmitted by grasp point $i$, denoted as $\mathbf{F}_{i}$ :

$$
\mathbf{F}_{i}=\mathbf{J}_{i}^{-T}\left(\boldsymbol{\Gamma}_{i}-\mathbf{A}_{i} \ddot{\mathbf{q}}_{i}-\mathbf{c}_{i}\right)
$$

If the serial arm contains passive joints, which have always zero torque, then the columns of $\mathbf{J}_{i}^{-T}$ that correspond to the actuated joint must be extracted resulting in a matrix $\mathbf{J}_{a i}^{-T}$ with dimension $\left(c_{i} \times a_{i}\right)$. Thus using (10), and (11), (12) becomes:

$$
\mathbf{F}_{i}=\mathbf{J}_{a i}^{-T} \boldsymbol{\tau}_{i}-\mathbf{A}_{x i} \dot{\mathbf{V}}_{i}-\mathbf{c}_{x i}
$$

where $\tau_{i}$ is a vector of $a_{i}$ components containing the actuated joint torques. Equation (13) relates the joint torques and the grasp wrench for the $i^{\text {th }}$ manipulator. 


\section{B. Flexible Object Equations}

The flexible object is modeled using free boundary conditions. A finite element analysis is carried out to obtain the values of $\boldsymbol{\Phi}_{d}(i)$, and $\boldsymbol{\Phi}_{r}(i)$ and the flexible inertial parameters. The object variables are related to the variables at the grasping points by a grasp matrix $\mathbf{W}$. The dimension of $\mathbf{W},(6 N \times 6 n)$, depends on the number of manipulators and the flexible coordinates. $\mathbf{W}$ is decomposed into $\mathbf{W}_{p}$, a $(6 \times 6 n)$ matrix, and $\mathbf{W}_{e}$ a $(N \times 6 n)$ matrix. Rewriting (4) for all manipulators:

$$
\left[\begin{array}{c}
\mathbf{V}_{1} \\
\vdots \\
\mathbf{V}_{n}
\end{array}\right]=\mathbf{L}^{T} \mathbf{W}^{T}\left[\begin{array}{c}
\mathbf{V}_{p} \\
\dot{\mathbf{q}}_{e}
\end{array}\right]=\mathbf{L}^{T}\left[\begin{array}{ll}
\mathbf{W}_{p}^{T} & \mathbf{W}_{e}^{T}
\end{array}\right]\left[\begin{array}{c}
\mathbf{V}_{p} \\
\dot{\mathbf{q}}_{e}
\end{array}\right]
$$

By differentiation:

$$
\left[\begin{array}{c}
\dot{\mathbf{V}}_{1} \\
\vdots \\
\dot{\mathbf{V}}_{n}
\end{array}\right]=\mathbf{L}^{T}\left(\mathbf{W}^{T}\left[\begin{array}{c}
\dot{\mathbf{V}}_{p} \\
\ddot{\mathbf{q}}_{e}
\end{array}\right]+\mathbf{h}\right)
$$

Similarly, from (7) and (14) it can be seen that:

$$
\left[\begin{array}{c}
\mathbf{F}_{p} \\
\mathbf{Q}_{p}
\end{array}\right]=\mathbf{W L}\left[\begin{array}{c}
\mathbf{F}_{1} \\
\vdots \\
\mathbf{F}_{n}
\end{array}\right]=\left[\begin{array}{c}
\mathbf{W}_{p} \\
\mathbf{W}_{e}
\end{array}\right] \mathbf{L}\left[\begin{array}{c}
\mathbf{F}_{1} \\
\vdots \\
\mathbf{F}_{n}
\end{array}\right]
$$

$\mathbf{W}$ is composed of a set of sub-matrices as follows:

$$
\mathbf{W}=\left[\begin{array}{c|c|c}
{ }^{1} \mathbf{S}_{p}^{T} & \ldots & { }^{n} \mathbf{S}_{p}^{T} \\
\boldsymbol{\Phi}^{T}(1) & \ldots & \boldsymbol{\Phi}^{T}(n)
\end{array}\right]=\left[\begin{array}{c|c|c}
\mathbf{W}_{p 1} & \ldots & \mathbf{W}_{p n} \\
\hdashline \mathbf{W}_{e 1} & \cdots & \mathbf{W}_{e n}
\end{array}\right]
$$

$\mathbf{L}$ is a $(6 n \times c)$ block diagonal matrix selection matrix, that projects the object variables into the connection point space, where the $i^{t h}$ component is $\mathbf{L}_{i}$, for $i=1 \ldots n . \mathbf{L}_{i}$ is a $\left(6 \times c_{i}\right)$ where each column represents a constraint direction. For example, $\mathbf{L}_{i}^{p}$ and $\mathbf{L}_{i}^{t y}$ that represent a planar and 1-DOF prismatic grasp condition respectively, are given as:

$$
\mathbf{L}_{i}^{p}=\left[\begin{array}{ccc}
1 & 0 & 0 \\
0 & 1 & 0 \\
0 & 0 & 0 \\
0 & 0 & 0 \\
0 & 0 & 0 \\
0 & 0 & 1
\end{array}\right] \quad \mathbf{L}_{i}^{t y}=\left[\begin{array}{l}
0 \\
1 \\
0 \\
0 \\
0 \\
0
\end{array}\right]
$$

\section{System Jacobian Matrix}

In this section the rigid arm variables and the flexible object variables are combined to derive two Jacobian matrices, denoted as $\mathbf{J}_{p}$ and $\mathbf{J}_{e}$. The matrix $\mathbf{J}_{p}^{-T}$ and $\mathbf{J}_{e}^{-T}$ relate the joint torques to the rigid and elastic object variables, respectively and are written as:

$$
\begin{aligned}
& \mathbf{J}_{p}^{-T}=\left[\begin{array}{lll}
\mathbf{W}_{p 1} \mathbf{L}_{1} \mathbf{J}_{a 1}^{-T} & \ldots & \mathbf{W}_{p n} \mathbf{L}_{n} \mathbf{J}_{a n}^{-T}
\end{array}\right] \\
& \mathbf{J}_{e}^{-T}=\left[\begin{array}{lll}
\mathbf{W}_{e 1} \mathbf{L}_{1} \mathbf{J}_{a 1}^{-T} & \ldots & \mathbf{W}_{e n} \mathbf{L}_{n} \mathbf{J}_{a n}^{-T}
\end{array}\right]
\end{aligned}
$$

where $\mathbf{J}_{p}^{-T}$ is $(6 \times a)$ matrix and $\mathbf{J}_{e}^{-T}$ is $(N \times a)$ matrix. Finally, the system Jacobian matrix is defined as :

$$
\mathbf{W}_{s}=\left[\begin{array}{l}
\mathbf{J}_{p}^{-T} \\
\mathbf{J}_{e}^{-T}
\end{array}\right]
$$

\section{System Resolution \& Identification}

The system is resolved by eliminating the variables at the grasping points in (13) by using (14), (16) and (19). In order to simplify the development, we denote $\mathbf{W}_{p} \mathbf{L}=\mathbf{W}_{p L}, \mathbf{W}_{e} \mathbf{L}=$ $\mathbf{W}_{e L}$ and $\mathbf{L}^{T} \mathbf{h}=\mathbf{h}_{L}$. Therefore, (13) can be rewritten as:

$$
\begin{aligned}
\mathbf{W}_{p L}\left[\begin{array}{c}
\mathbf{F}_{1} \\
\vdots \\
\mathbf{F}_{n}
\end{array}\right] & =\mathbf{J}_{p}^{-T} \boldsymbol{\tau} \\
& -\mathbf{W}_{p L}\left(\mathbf{A}_{x}\left(\mathbf{W}_{p L}^{T} \dot{\mathbf{V}}_{p}+\mathbf{W}_{e L}^{T} \ddot{\mathbf{q}}_{e}+\mathbf{h}_{L}\right)+\mathbf{c}_{x}\right) \\
\mathbf{W}_{e L}\left[\begin{array}{c}
\mathbf{F}_{1} \\
\vdots \\
\mathbf{F}_{n}
\end{array}\right] & =\mathbf{J}_{e}^{-T} \boldsymbol{\tau} \\
& -\mathbf{W}_{e L}\left(\mathbf{A}_{x}\left(\mathbf{W}_{e L}^{T} \dot{\mathbf{V}}_{p}+\mathbf{W}_{e L}^{T} \ddot{\mathbf{q}}_{e}+\mathbf{h}_{L}\right)+\mathbf{c}_{x}\right)
\end{aligned}
$$

where $\mathbf{A}_{x}$ is a $(c \times n) \times(c \times n)$ block diagonal matrix where the $i^{\text {th }}$ block is $\mathbf{A}_{x i}$. $\mathbf{c}_{x}$ is an $c \times 1$ vector such that $\mathbf{c}_{x}=$ $\left[\begin{array}{lll}\mathbf{c}_{x 1} & \ldots & \mathbf{c}_{x 6}\end{array}\right]^{T}$. The dynamic model (8) of the object is rewritten in simpler form as:

$$
\left[\begin{array}{l}
\mathbf{W}_{p L} \\
\mathbf{W}_{e L}
\end{array}\right]\left[\begin{array}{l}
\mathbf{F}_{1} \\
\vdots \\
\mathbf{F}_{6}
\end{array}\right]=\left[\begin{array}{cc}
\mathbf{A}_{r} & \mathbf{A}_{r e} \\
\mathbf{A}_{r e}^{T} & \mathbf{m}_{e e}
\end{array}\right]\left[\begin{array}{l}
\dot{\mathbf{V}}_{p} \\
\ddot{\mathbf{q}}_{e}
\end{array}\right]+\left[\begin{array}{l}
\mathbf{c}_{r} \\
\mathbf{c}_{e}
\end{array}\right]
$$

Finally, by equating (21), (22) and (23), the system dynamics are obtained as:

$$
\begin{aligned}
\mathbf{W}_{s} \boldsymbol{\tau} & =\left[\begin{array}{cc}
\mathbf{A}_{r}+\mathbf{W}_{p L} \mathbf{A}_{x} \mathbf{W}_{p L}^{T} & \mathbf{A}_{r e}+\mathbf{W}_{p L} \mathbf{A}_{x} \mathbf{W}_{e L}^{T} \\
\mathbf{A}_{r e}^{T}+\mathbf{W}_{e L} \mathbf{A}_{x} \mathbf{W}_{p L}^{T} & \mathbf{m}_{e e}+\mathbf{W}_{e L} \mathbf{A}_{x} \mathbf{W}_{e L}^{T}
\end{array}\right]\left[\begin{array}{c}
\dot{\mathbf{V}}_{p} \\
\ddot{\mathbf{q}}_{e}
\end{array}\right] \\
+ & {\left[\begin{array}{c}
\mathbf{c}_{r}+\mathbf{W}_{p L}\left(\mathbf{A}_{x} \mathbf{h}_{L}+\mathbf{c}_{x}\right) \\
\mathbf{c}_{e}+\mathbf{W}_{e L}\left(\mathbf{A}_{x} \mathbf{h}_{L}+\mathbf{c}_{x}\right)
\end{array}\right] }
\end{aligned}
$$

The matrix $\mathbf{W}_{s}$ has dimension $(6+N \times a)$. In the following section, the closed chain system is analyzed by examining this matrix, which in turn leads to an object classification.

\section{CAse Studies}

A solution for three classes of objects are given for the cooperative system shown in Fig.3. The objective is to validate the model using a commercial dynamic simulator as shown as shown in Fig.2. Since the system is planar $\operatorname{dim}\left(\dot{\mathbf{V}}_{p}\right)=3$. The system is described using modified Denavit and Hartenberg notations [24] given in Table I. The non-zero base inertial parameters are given as:

$$
\begin{array}{rlrlrl}
Z Z_{1} & =5.0 \mathrm{kgm}^{2} & Z Z_{2} & =5.0 \mathrm{kgm}^{2} & Z Z_{3} & =4.0 \mathrm{kgm}^{2} \\
M_{1} & =10.0 \mathrm{~kg} & M_{2} & =5.0 \mathrm{~kg} & M_{3} & =5.0 \mathrm{~kg}
\end{array}
$$

\section{A. Case 1: Rigid Object}

The object is rigid i.e. $\mathbf{q}_{e}=\dot{\mathbf{q}}_{e}=\ddot{\mathbf{q}}_{e}=0$. The system is simulated with 6 actuated joints. Therefore $\mathbf{W}_{s}=\mathbf{J}_{p}^{-T}$ is a $(3 \times 6)$ matrix. 
TABLE I. GEOMETRIC PARAMETERS FOR ARM $i$

\begin{tabular}{cccccccc}
\hline \hline$j_{i}$ & $\sigma_{j i}$ & $\gamma_{j i}$ & $b_{j i}$ & $\alpha_{j i}$ & $d j i$ & $\theta_{j i}$ & $r_{j i}$ \\
\hline $1_{i}$ & 0 & 0 & 0 & 0 & 0 & $q_{1 i}$ & 0 \\
$2_{i}$ & 0 & 0 & 0 & 0 & 0.5 & $q_{2 i}$ & 0 \\
$3_{i}$ & 0 & 0 & 0 & 0 & 0.5 & $q_{3 i}$ & 0 \\
$4_{i}$ & 2 & 0 & 0 & 0 & 0.2 & 0 & 0 \\
\hline \hline
\end{tabular}

1) Solution: The solution is identical to that of a parallel robot with rigid legs described in [25], but represented in the Cartesian space:

$$
\mathbf{W}_{s} \boldsymbol{\tau}=\left(\mathbf{A}_{r}+\mathbf{W}_{p L} \mathbf{A}_{x} \mathbf{W}_{p L}^{T}\right) \dot{\mathbf{V}}_{p}+\mathbf{c}_{r}+\mathbf{W}_{p L}\left(\mathbf{A}_{x} \mathbf{h}_{L}+\mathbf{c}_{x}\right)
$$

which is rewritten as $\mathbf{W}_{s} \boldsymbol{\tau}=\mathbf{F}_{\text {motion }}$. From the structure of $\mathbf{W}_{s}$, it is clear that the system is redundantly actuated, thus the object may undergo internal loading. By definition, the internal loading has no effect on the motion causing forces. Therefore inverting (25) results in the classical formulation for cooperative manipulators [15]:

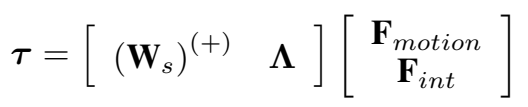

where $\Lambda$ may be any matrix that spans the null space of $\mathbf{W}_{s}$ and $\mathbf{F}_{\text {int }}$ denotes the internal forces.

2) Validation: The Cartesian acceleration, $\dot{\mathbf{V}}_{p}$, and internal loading are calculated from the joint torque and robot's state. The object's calculated Cartesian acceleration is compared with the Adams equivalent in Fig.4. The error of the internal forces is given in Fig.5. In both cases a good correlation is seen.

\section{B. Case 2: Flexible Object}

The object is flexible; the elastic variables cannot be controlled, but may be damped by a judicious choice of controller. The system contains 3 actuated joints and the object is modeled using 22 shape functions i.e. 22 elastic variables.

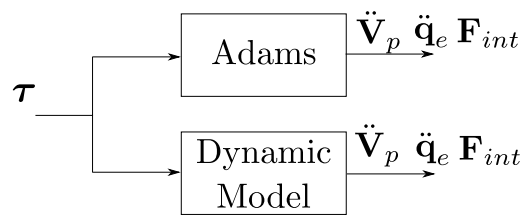

Fig. 2. Validation Procedure of Dynamic Model

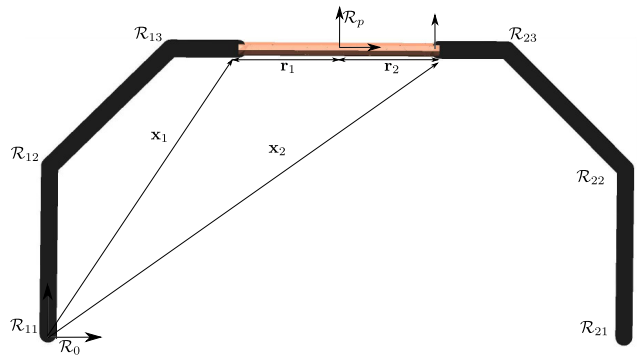

Fig. 3. Dual arm robots with object
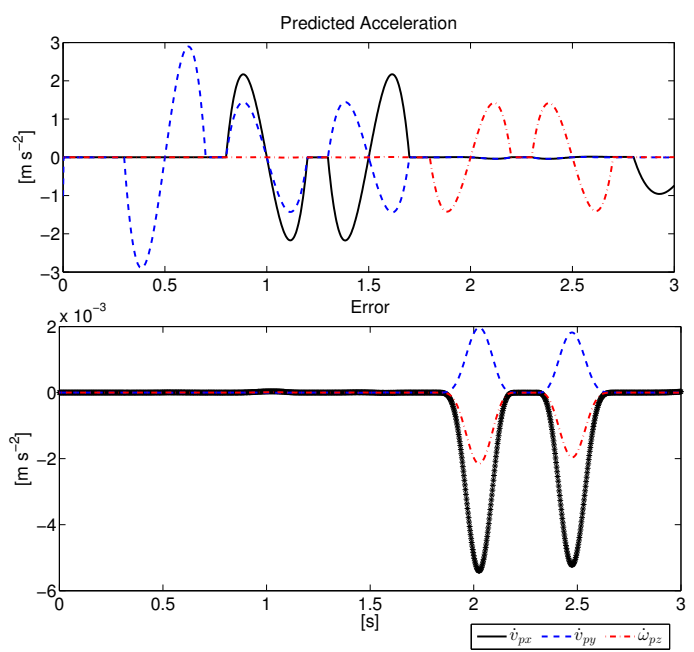

Fig. 4. Rigid Object Acceleration: (Top) Predicted acceleration of object, (Bottom) Difference between Adams and predicted model

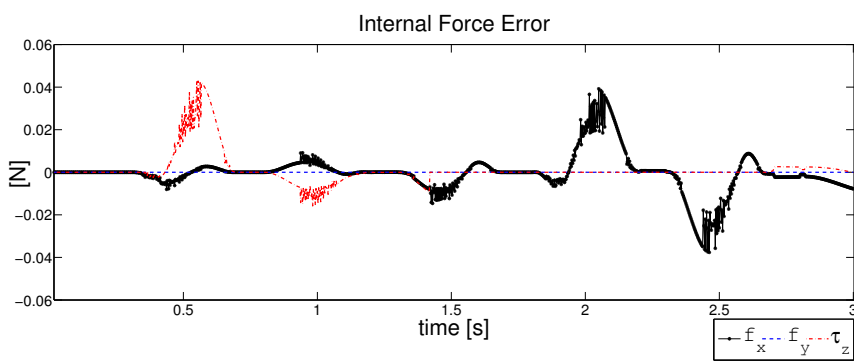

Fig. 5. Rigid Object Forces: Difference between Adams and predicted model for desired internal force $\mathbf{F}_{\text {int }}=\left[\begin{array}{lll}0 & 100 & 0\end{array}\right]$
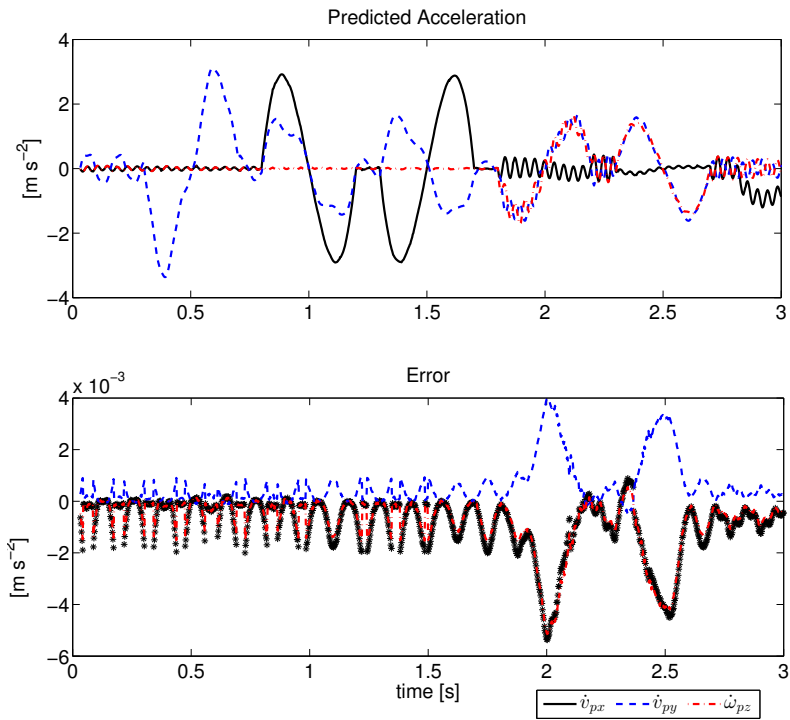

Fig. 7. Flexible Object: (Top) Predicted Acceleration of flexible object, (Bottom) Difference between Adams and predicted model

1) Solution: $\mathbf{W}_{s}$ is a $(28 \times 3)$ matrix that cannot be inverted, thus a 2-step solution is required. Firstly, using the second row 

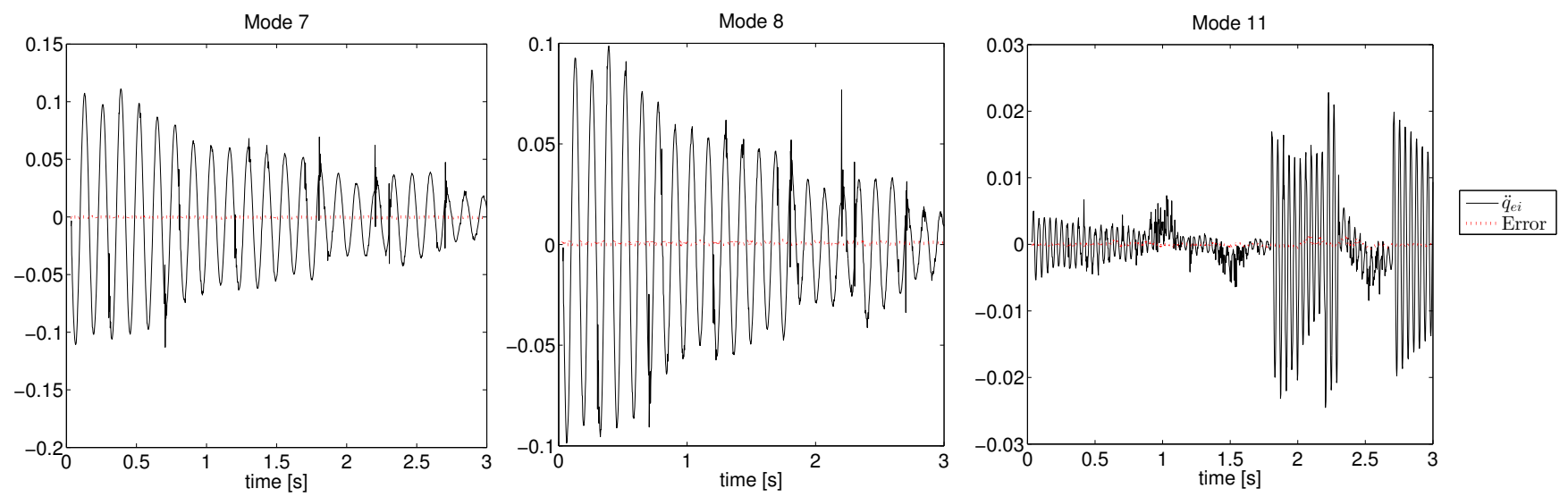

Fig. 6. Flexible Object: Error between predicted \& measured acceleration for high magnitude modes

of (24) the elastic variables are rewritten as:

$$
\begin{gathered}
\ddot{\mathbf{q}}_{e}=\mathbf{A}_{e e}^{-1}\left(\mathbf{J}_{e}^{-T} \boldsymbol{\tau}-\left(\mathbf{A}_{r e}^{T}+\mathbf{W}_{e L} \mathbf{A}_{x} \mathbf{W}_{p L}^{T}\right) \dot{\mathbf{V}}_{p}-\mathbf{W}_{e L} \mathbf{A}_{x} \mathbf{h}_{L}\right) \\
-\mathbf{A}_{e e}^{-1}\left(\mathbf{W}_{e L} \mathbf{c}_{x}-\mathbf{c}_{e}\right)
\end{gathered}
$$

where for convenience we define $\mathbf{A}_{e e}^{-1}=$ $\left(\mathbf{m}_{e e}+\mathbf{W}_{e L} \mathbf{A}_{x} \mathbf{W}_{e L}^{T}\right)^{-1}$. Secondly, (27) is back-substituted into (24) to obtain the dynamic model of the manipulator:

$$
\mathbf{A} \dot{\mathbf{V}}_{p}+\mathbf{c}=\mathbf{J}_{s y s}^{-T} \boldsymbol{\tau}
$$

The $6 \times 6$ matrix $\mathbf{A}$ is the equivalent total inertia matrix of the legs and the flexible object is written as:

$$
\begin{aligned}
\mathbf{A}= & \mathbf{A}_{r r}+\mathbf{W}_{p L} \mathbf{A}_{x} \mathbf{W}_{p L}^{T}-\mathbf{A}_{r e} \mathbf{A}_{e e}^{-1}\left(\mathbf{A}_{r e}^{T}+\mathbf{W}_{e L} \mathbf{A}_{x} \mathbf{W}_{p L}^{T}\right) \\
& -\mathbf{W}_{p L} \mathbf{A}_{x} \mathbf{W}_{e L}^{T} \mathbf{A}_{e e}^{-1}\left(\mathbf{A}_{r e}^{T}+\mathbf{W}_{e L} \mathbf{A}_{x} \mathbf{W}_{p L}^{T}\right)
\end{aligned}
$$

The $6 \times 6$ Jacobian matrix is given by

$$
\mathbf{J}_{s y s}^{-T}=\mathbf{J}_{p}^{-T}-\left(\mathbf{A}_{r e}+\mathbf{W}_{p L} \mathbf{A}_{x} \mathbf{W}_{e L}^{T}\right) \mathbf{A}_{e e}^{-1} \mathbf{J}_{e}^{-T}
$$

The $6 \times 1$ vector $\mathbf{c}$, the total Coriolis, centrifugal and gravity torques of the legs and the flexible object, is given as:

$$
\begin{aligned}
\mathbf{c}= & \mathbf{c}_{r}+\mathbf{W}_{p L} \mathbf{A}_{x} \mathbf{h}_{L}+\mathbf{W}_{p L} \mathbf{c}_{x}- \\
& \left(\mathbf{A}_{r e}+\mathbf{W}_{p L} \mathbf{A}_{x} \mathbf{W}_{e L}^{T}\right) \mathbf{A}_{e e}^{-1}\left(\mathbf{W}_{e L} \mathbf{A}_{x} \mathbf{h}+\mathbf{W}_{e L} \mathbf{c}_{x}+\mathbf{c}_{e}\right)
\end{aligned}
$$

2) Validation: To validate the model the Cartesian acceleration is obtained by inverting the positive-definite total inertia matrix to solve (28). Once $\dot{\mathbf{V}}_{p}$ is obtained the generalized elastic variables are calculated from (27).

Fig. 7 shows a small errors between the ADAMS output and the calculated Cartesian accelerations despite large vibrations. Fig.6 compares the ADAMS output to the calculated values of the generalized elastic variables' acceleration, thus demonstrating how the model can accurately predict vibration in the system.

\section{Case 3: Articulated Object}

There are sufficient actuators to fully control the object's state. The system contains 6 actuated joints and the object has a degree of flexibility of dimension 3 .
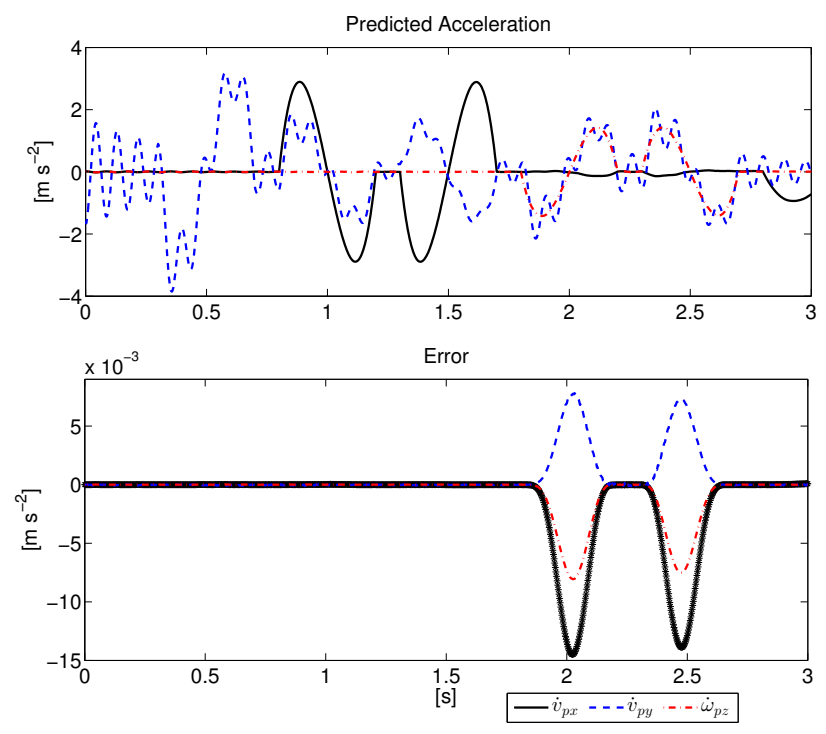

Fig. 9. Articulated Object: (Top) Predicted Acceleration of flexible object, (Bottom) Difference between Adams and predicted model

1) Solution: $\mathbf{W}_{s}$ has full rank. From examination of (24), it is clear that by inverting $\mathbf{W}_{s}$, joint torques can be obtained that allow the system to perfectly achieve a desired rigid body and elastic generalized acceleration. For this type of system, the vibration induced by the flexibility can be perfectly suppressed.

2) Validation: To validate the model the Cartesian acceleration and the generalized elastic variables are calculated from the joint torques directly by solving (24). Fig.9 gives a comparison between the ADAMS output and the calculated Cartesian accelerations. Fig.8 displays a comparison between the ADAMS output and the calculated of the generalized elastic variables' acceleration.

\section{CONCLUSIONS}

This work has outlined a general strategy for modeling cooperative manipulators grasping common objects. The formulation is founded on the decomposition of the system into a rigid and flexible subsystems allowing the variables of each to be calculated separately. A closed form solution for the 

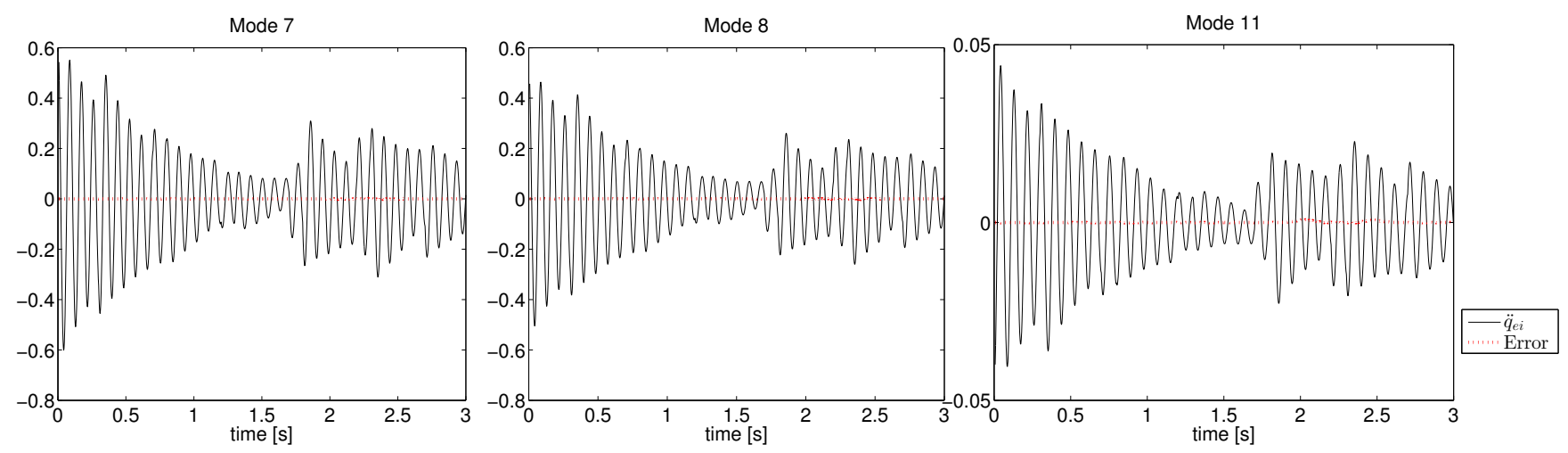

Fig. 8. Articulated Object: Error between predicted \& measured acceleration for active modes

dynamic model is obtained which, depending on the object, can be resolved in a number of ways. For three different object types, the dynamic model has been compared with a commercial system and the validity of the results have been shown.

The principal advantages of the solution proposed in this paper, with respect to the numerical solution in the simulation software, are computation speed and ease of implementation. In addition to this, the closed form solution can be exploited to generate a series of computed torque control schemes. Future work will focus on the design of these control schemes and the experimental validation of the algorithm.

\section{REFERENCES}

[1] K. Cleary and C. Nguyen, "State of the art in surgical robotics: clinical applications and technology challenges," Computer Aided Surgery, vol. 6 , no. 6 , pp. 312-328, 2001.

[2] R. J. M. Masey, J. O. Gray, T. J. Dodd, and D. G. Caldwell, "Guidelines for the design of low-cost robots for the food industry," Industrial Robot: An International Journal, vol. 37, no. 6, pp. 509-517, 2010.

[3] D. Navarro-Alarcón, Y.-H. Liu, J. G. Romero, and P. Li, "Modelfree visually servoed deformation control of elastic objects by robot manipulators," IEEE Transactions on Robotics and Automation, vol. 29, pp. 1457-1468, dec 2013.

[4] P. Long, W. Khalil, and P. Martinet, "Force/vision control for robotic cutting of soft materials," in 2014 IEEE/RSJ International Conference on Intelligent Robots and Systems (IROS), 2014, 2014.

[5] D. Sun and Y.-h. Liu, "Position and Force Tracking of a System Manipulating a Flexible Beam," Journal of Robotic Systems, vol. 18, pp. 197-212, 2001.

[6] P. Long, W. Khalil, and P. Martinet, "Dynamic modeling of parallel robots with flexible platforms," Mechanism and Machine Theory, vol. 81, pp. 21-35, 2014.

[7] M. Nahon and J. Angeles, "Force optimization in redundantly-actuated closed kinematic chains," in Proceedings of the 1989 IEEE International Conference on Robotics and Automation, pp. 951-956, 1989.

[8] A. Muller, "Internal preload control of redundantly actuated parallel manipulators its application to backlash avoiding control," IEEE Transactions on Robotics, vol. 21, no. 4, pp. 668-677, 2005.

[9] P. Long, W. Khalil, and S. Caro, "Kinematic and dynamic analysis of lower-mobility cooperative arms," Robotica, vol. FirstView, pp. 1-22, 52014.

[10] F. Caccavale, P. Chiacchio, A. Marino, and L. Villani, "Six-DOF impedance control of dual-arm cooperative manipulators," IEEE/ASME Transactions on Mechatronics, vol. 13, no. 5, pp. 576-586, 2008.

[11] J. Das and N. Sarkar, "Autonomous shape control of a deformable object by multiple manipulators," Journal of Intelligent \& Robotic Systems, vol. 62 , no. 1, pp. 3-27, 2011.
[12] W. F. Dellinger and J. Anderson, "Interactive force dynamics of two robotic manipulators grasping a non-rigid object," in Robotics and Automation, 1992. Proceedings., 1992 IEEE International Conference on, pp. 2205-2210 vol.3, 1992.

[13] M. Uchiyama and P. Dauchez, "A symmetric hybrid position/force control scheme for the coordination of two robots," in Proceedings of the 1988 IEEE International Conference on Robotics and Automation, Philadelphia, PA, pp. 350-356, 1988.

[14] S. Hayati, "Hybrid position/force control of multi-arm cooperating robots," in Proceedings of the 1986 IEEE International Conference on Robotics and Automation, vol. 3, pp. 82-89, apr 1986.

[15] R. C. Bonitz and T. C. Hsia, "Internal force-based impedance control for cooperating manipulators," IEEE Transactions on Robotics and Automation, vol. 12, no. 1, pp. 78-89, 1996.

[16] A. Tavasoli, M. Eghtesad, and H. Jafarian, "Two-time scale control and observer design for trajectory tracking of two cooperating robot manipulators moving a flexible beam," Robot. Auton. Syst., vol. 57, pp. 212-221, Feb. 2009.

[17] A. S. Al-Yahmadi, J. Abdo, and T. Hsia, "Modeling and control of two manipulators handling a flexible object," Journal of the Franklin Institute, vol. 344, no. 5, pp. 349 - 361, 2007. Modeling, Simulation and Applied Optimization Part II

[18] S. Dong and L. Yunhui, "Modeling and impedance control of a twomanipulator system handling a flexible beam," in Proceedings of 1997 IEEE International Conference on Robotics and Automation (ICRA), vol. 2, pp. 1787-1792 vol.2, 1997.

[19] D. Weer and S. Rock, "Experiments in object impedance control for flexible objects," in Proceedings of the 1994 IEEE International Conference on Robotics and Automation, (ICRA), pp. 1222-1227 vol.2, 1994.

[20] T. Wada, S. Hirai, S. Kawamura, and N. Kamiji, "Robust manipulation of deformable objects by a simple pid feedback," in Robotics and Automation, 2001. Proceedings 2001 ICRA. IEEE International Conference on, vol. 1, pp. 85-90, 2001.

[21] F. Boyer and W. Khalil, "An efficient calculation of flexible manipulator inverse dynamics," The International Journal of Robotics Research, vol. 17, no. 3, pp. 282-293, 1998.

[22] F. Boyer, W. Khalil, M. Benosman, and G. Le Vey, "Modeling and control of flexible robots," in Robot Manipulators: Modeling, Performance Analysis and Control (E. Dombre and W. Khalil, eds.), ch. 7, pp. 337-394, Cambridge, Massachusetts: ISTE Ltd, 2007.

[23] O. Khatib, "A unified approach for motion and force control of robot manipulators: The operational space formulation," IEEE Journal of Robotics and Automation, vol. 3, no. 1, pp. 43-53, 1987.

[24] W. Khalil and J. Kleinfinger, "A new geometric notation for open and closed-loop robots," in Robotics and Automation. Proceedings. 1986 IEEE International Conference on, vol. 3, pp. 1174-1179, IEEE, 1986.

[25] W. Khalil and O. Ibrahim, "General solution for the dynamic modeling of parallel robots," Journal of Intelligent and robotic systems, vol. 49, no. 1 , pp. 19-37, 2007. 